\title{
Reconsolidation after remembering an odor-reward association requires NMDA receptors
}

\author{
Meritxell Torras-Garcia, Julien Lelong, Sophie Tronel, and Susan J. Sara ${ }^{1}$ \\ Laboratory of Neuromodulation and Memory, CNRS UMR 7102, Université Pierre \& Marie Curie, 75005 Paris, France
}

\begin{abstract}
A rapidly learned odor discrimination task based on spontaneous foraging behavior of the rat was used to evaluate the role of N-methyl-D-aspartate (NMDA) receptors (NMDARs) in ongoing memory consolidation. Rats were trained in a single session to discriminate among three odors, one of which was associated with palatable food reward. Previous experiments showed that the NMDAR antagonist DL-APV induced amnesia for this task when injected immediately after training. In the present study, memory was reactivated $24 \mathrm{~h}$ after training by exposure to the rewarded odor within the experimental context after which rats received an intracerebroventricular injection of APV. Combined reactivation-drug treatment induced profound amnesia when tested $48 \mathrm{~h}$ later. Animals receiving drug alone, in absence of reactivation, showed perfect retention. It is concluded that NMDARs support a consolidation process taking place after memory reactivation.
\end{abstract}

A marked conceptual change in the neurobiological view of memory consolidation processes is emerging. For most of the twentieth century, a general consensus prevailed that memory traces are consolidated over a short time after the initial experience and then remain stable. Earlier philosophers and psychologists often held a more dynamic view of memory in a constant state of flux (James 1890; Bartlett 1932), maintaining that each time a memory is retrieved, it is integrated into ongoing perceptual and emotional experiences and becomes part of a new memory. Furthermore, the new memory requires reconsolidation to be returned to a stable form (see Sara 2000 for a review of early studies). Recent studies have provided evidence for this by showing that well consolidated memories, recalled by way of a reminder, become vulnerable to amnesic agents (for review, see Dudai 2004). It appears, moreover, that initial consolidation and reconsolidation are qualitatively similar processes across species, in that some of the critical cellular events in consolidation are also necessary for reconsolidation. These events include synthesis of new RNA (Sangha et al. 2003) and proteins (Nader et al. 2000; Taubenfeld et al. 2001; Anokhin et al. 2002; Debiec et al. 2002; Kida et al. 2002; Milekic and Alberini 2002; Pedreira et al. 2002; Sangha et al. 2003), and activation of the MAP kinase pathway (Kelly et al. 2003), the cAMP response element-binding protein (CREB) (Hall et al. 2001; Kida et al. 2002), and immediate early genes c-fos (Hall et al. 2001) and zif268 (Hall et al. 2001). As the list suggests, the focus has been on possible intracellular common pathways that might be common to consolidation and reconsolidation. Less is known about synaptic events initiating the cascades leading to new protein synthesis after remembering, and even less still, about the neural circuits involved.

Tsien and colleagues have suggested a unique role for $\mathrm{N}$ methyl-D-aspartate (NMDA) receptors (NMDARs) in maintaining memory after initial consolidation, proposing a "synaptic reentry reinforcement" hypothesis. According to this view, neuronal ensembles involved in initial learning are continually reactivated and undergo "multiple rounds" of synaptic reinforcement. This synaptic reinforcement, occurring over several days, is NMDARdependent, so that the NMDARs within the circuit-to-bereinforced would be periodically reactivated, to reinitiate the

'Corresponding author.

E-mail sjsara@ccr.jussieu.fr; fax 33-1-4427-3252.

Article published online ahead of print. Article and publication date are at http://www.learnmem.org/cgi/doi/10.1101/lm.80905. consolidation process (reconsolidation) (Shimizu et al. 2000; Wittenberg and Tsien 2002). The reactivation paradigm now being widely used to evaluate the neurobiological processes involved in reconsolidation can make a first step in addressing this question by evaluating the role of NMDARs in reconsolidation after a reactivation.

Most reactivation-reconsolidation studies have been carried out using single-trial aversive paradigms, notably inhibitory avoidance and conditioned fear. The few exceptions include one of the first demonstrations of memory lability after reactivation, made by Lewis et al. (1972), using an appetitively motivated linear maze task. Przybyslawski and Sara (1997) and Przybyslawski et al. (1999) later showed that memory became labile after reactivation of a radial arm maze task that was food-reinforced. These tasks have the advantage of reliable behavioral performance and minimal stress. Nevertheless, they are of limited use in directly comparing consolidation and reconsolidation processes, because they are acquired in multiple trials, usually over several daily sessions. Some memory consolidation presumably takes place after each session; thus any amnestic treatment would have to be applied after each session to interfere with consolidation, while a reconsolidation protocol requires only one single amnestic treatment, after the reactivation of the memory that was acquired gradually over several sessions. In the present experiment we used a rapidly acquired appetitive odor discrimination task, in which the essential role of NMDARs in initial consolidation has been clearly established (Tronel and Sara 2003). The task can thus be used to study the role of NMDARs in reconsolidation, addressing the question of whether some cellular processes underlying reconsolidation recapitulate those of initial consolidation.

\section{Results}

\section{Task acquisition}

Rats were trained on the three-way odor discrimination in three massed trials. Rapid learning was attested to by the progressive decrease in latency to find the correct sponge and make the nose poke response; moreover, rats rarely made a nose-poke error by the third trial.

\section{Test and relearning}

The test phase, $48 \mathrm{~h}$ after training, consisted of four trials, the first of which was not rewarded. The mean latency for each group 
A
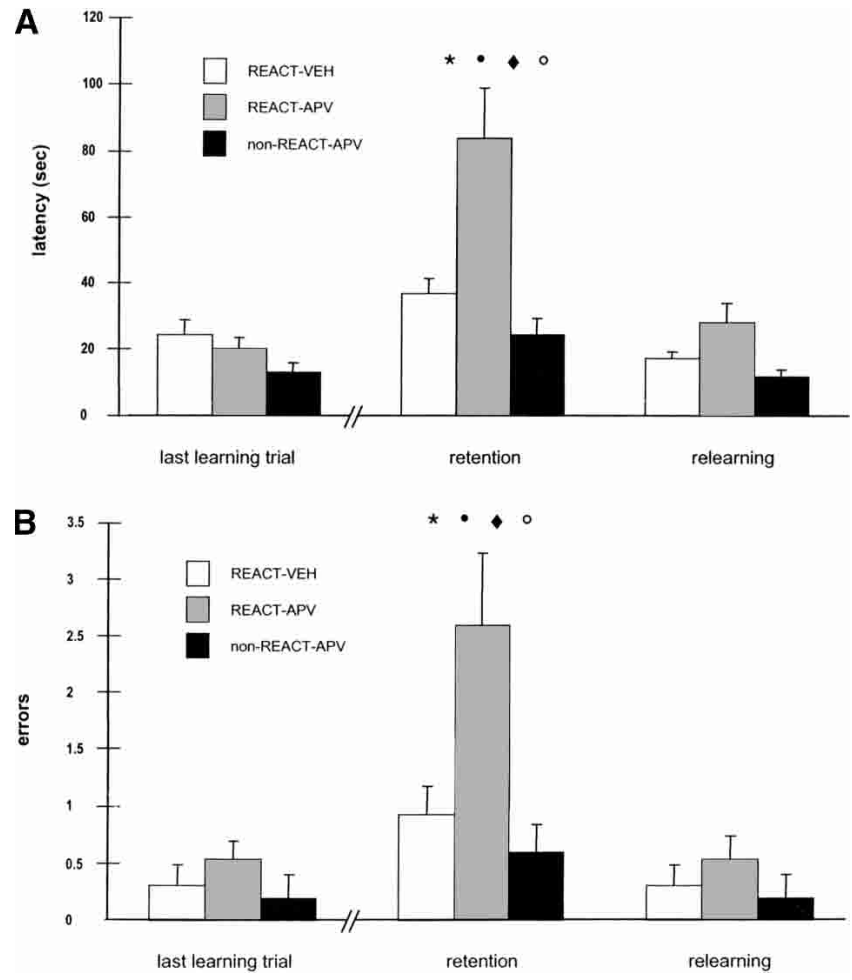

Figure 1. (A) Latency to make the correct response over the last training trial, the retention, and the relearning. APV-REACT is significantly different from REACT-VEH $\left({ }^{*}, P=0.005\right)$ and NON-REACT-APV $(\bullet$, $P=0.009)$ at the retention test. Significant differences were observed between the retention test and the last learning trial $(O, P=0.001)$ and between the retention test and the relearning $(\diamond, P<0.001)$ for REACTAPV. (B) Errors during the last training trial, retention and relearning. APV-REACT is significantly different from REACT-VEH $(*, P=0.02)$ and NON-REACT-APV $(\bullet, P=0.04)$. Significant differences were observed between retention and last learning trial $(O, P=0.003)$ and between retention and relearning $(\diamond, P<0.001)$ for the REACT-APV group.

on the last training trial, the two test trials, and the two relearning trials can be seen in Figure 1A. ANOVA for repeated measures was applied to these data, one factor being group (three groups: REAC-VEH, $n=13$, REACT-APV, $n=13$, and nonREACT-APV, $n=5$ ); the repeated factor being training, retention, and relearning. ANOVA revealed that a main effect, group and the group $\times$ trial interaction were highly significant $\left(F_{(1,30)}=6.95\right.$, $P=0.003 ; F_{(4,60)}=4.45, P=0.003$, respectively). As shown in Figure $1 \mathrm{~A}, \mathrm{APV}$ administered immediately after the reactivation session produced a performance decrement at the test trial. Planned post hoc comparisons revealed a significant difference between the REACT-APV and both the REACT-VEH $(P=0.005)$ and nonREACT-APV $(P=0.009)$ groups at the retention test. Significant differences were also observed between the last trial of training and the retention test $(P=0.001)$ and between the retention test and the relearning $(P<0.001)$ for the REACT-APV group. Together, these data indicate that blockade of NMDARs immediately after reactivation induces amnesia but does not block the relearning.

A similar pattern of results is seen when errors are considered as the performance measure (Fig. 1B). The data were analyzed as described above for latencies. Here the main effect group was significant $\left(F_{(2,30)}=9.89 ; P=0.011\right)$ and the interaction group $\times$ trial approached significance $\left(F_{(4,60)}=2.37 ; P=0.062\right)$. Planned comparisons showed that REACT-APV made significantly more errors at the test than REACT-VEH $(P=0.02)$ and nonREACT-APV $(P=0.04)$. Moreover, the within-group differ- ences between last training trial and retention test and between retention test and relearning were also significant for REACTAPV only $(P=0.003$ and $P<0.001$, respectively).

Complementary analysis was conducted with the parameter "path distance" calculated using EthoVision software. Figure 2A shows the path for each individual rat at the first retention test. Figure 2B shows the group mean distance traveled on the last training trial, retention trials, and relearning trials. These data were analyzed as described above for latencies and errors. Contrast analysis showed that REACT-APV was the only group that traveled a greater distance at retention session $(P=0.009)$ than at the last training session, and also differs from the REACT-VEH in the test session $(P=0.03)$.

\section{Discussion}

\section{NMDA receptors support reconsolidation after reactivation}

The results provide clear evidence that NMDARs are involved in memory consolidation processes taking place long after the initial acquisition. The NMDAR antagonist DL-APV injected into the lateral ventricles $24 \mathrm{~h}$ after training induces a profound and enduring amnesia for a well learned olfactory memory. This effect cannot be due to a pro-active, nonspecific effect $48 \mathrm{~h}$ after the treatment, since APV alone, administered in a neutral context, does not have any effect on retention performance. It is only effective in rats that have been exposed to a reminder, right before the injection. There is always the possibility that the association of the drug treatment with the odor could produce some aversion that would account for the behavioral effects at the retention test. This is unlikely, since the rats show rapid relearning after reinforced trials during the test. Moreover, we have shown in previous studies that blockade of NMDA receptors with systemic injections of MK801 in a dose range that produces amnesia does not induce conditioned aversion (Pryzbyslawski and Sara 1997). Another possible confounding variable could be faster extinction induced by the combination of unreinforced $\mathrm{CS}+$ exposure and subsequent drug treatment. However, in numerous pilot studies examining the efficacy of different reactivation protocols, we never observed any tendency towards extinction with off-line exposure of the CS+, $48 \mathrm{~h}$ before testing.

Our results are consistent with the view, originally put forth by Lewis et al. (1972) that a consolidated memory retrieved by a reminder passes from an inactive and stable state, to an active and labile state that undergoes a memory reconsolidation process to be returned to a stable form. NMDA receptors appear to be necessary for this reconsolidation process, as they have been shown to be in initial consolidation for the same task (Tronel and Sara 2003). It should be noted however that while DL-APV binds preferentially to NMDA receptors, there is some nonspecific binding that could contribute to the behavioral deficit. This is unlikely, because rats receiving the drug treatment alone showed no such deficit.

The results further support the view that NMDARs are engaged for an extended time period after initial acquisition and memory consolidation (Shimizu et al. 2000). On the other hand, the fact that a specific reminder procedure is a necessary condition to induce amnesia does not support the view that the ongoing reconsolidation is due to random reactivation of the hippocampal network (Wittenberg and Tsien 2002), but rather supports these authors' alternative suggestion that it could be triggered by "conscious recall." The retrieval cue used in the present experiment is a brief exposure to the holding cage in the experimental room (context) and the odor associated with the reward during training (CS+). Other retrieval cues might be as 
A

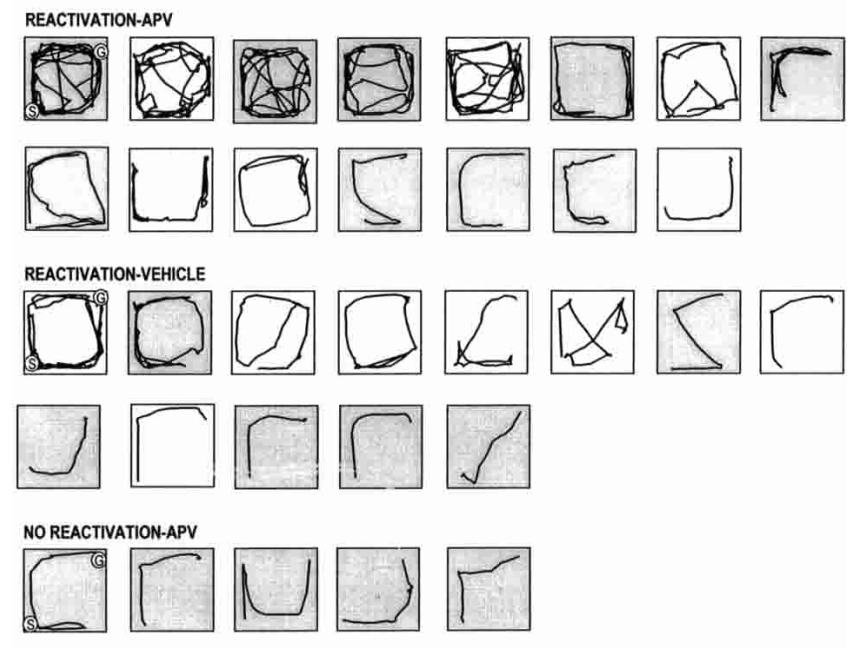

B

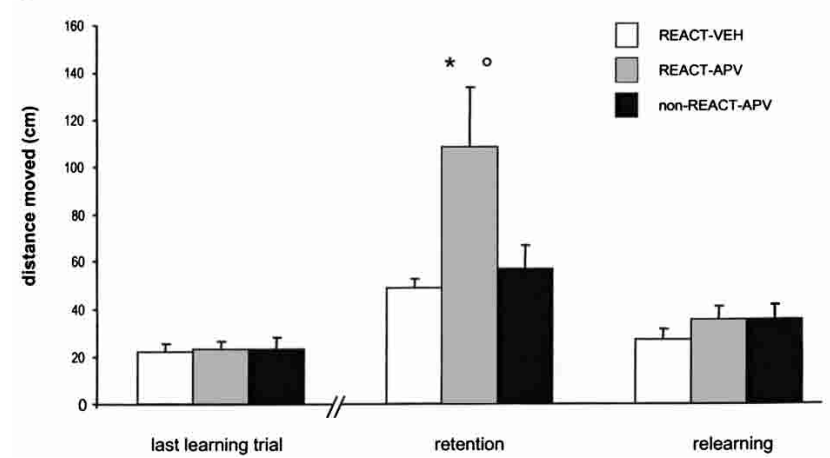

Figure 2. (A) Path of each rat on the first retention trial. Note that the REACT-APV group, showing amnesia in terms of latency and errors, shows more search behavior at the retention test. G, Goal; S, Start. The Goal and Start positions were the same for each rat on a given trial and were changed at each successive trial. $(B)$ Distance traveled during the last training trial, retention, and relearning. REACT-APV was significantly different from REACT-VEH $(*, P=0.03)$. Significant differences between retention and last learning trial $(O, P=0.009)$

effective as the combination used here-for example, the context alone or in combination with the nontarget odors. The presentation of retrieval cues whatever they be, might be the closest we can get to inducing "conscious recall" in the rat.

\section{The behavioral task}

The seminal studies by Lewis et al. (1972) used an appetitively motivated, multisession linear maze task to initially demonstrate the lability of active memory, by applying electroconvulsive shock after a reminder. Later studies suggested a role for NMDARs in reconsolidation after reactivation of a memory for a foodreinforced radial arm maze task acquired across multiple sessions (Przybyslawski and Sara 1997). Using the same protocol it was shown that $\beta$-adrenergic receptors were implicated in a late phase of reconsolidation (Roullet and Sara 1998; Przybyslawski et al. 1999). These multisession protocols, in which reliable, stable performance is achieved over several hours or days, have thus proved to be well suited to study time-dependent reconsolidation processes, since the memory reactivation (retrieval) occurs as a discrete event in temporal contiguity with reminder. On the other hand, the study of initial consolidation processes requires rapidly learned tasks where acquisition can be pinpointed in time, or alternatively, multiple amnestic treatments occurring in temporal contiguity with each trial. Thus the important question of whether consolidation and reconsolidation are qualitatively similar processes requiring the same or similar critical cellular events cannot easily be addressed using learning paradigms requiring multiple acquisition sessions, because of lack of symmetry between post-acquisition and post-reactivation treatments. For this reason most of the research directed at revealing cellular processes of reconsolidation are currently carried out using single-trial aversive learning such as inhibitory avoidance and conditioned fear. These behavioral protocols necessarily involve a high level of stress and rely almost exclusively on the conditioned emotional response of immobility. Memory lability after reactivation could conceivably be particular to highly emotional fear memories. Therefore it is important to include in these studies other behavioral tasks that are not based on conditioned fear. The olfactory discrimination task based on foraging for palatable food used in the present study fulfills the requirement for rapid acquisition in a single, three-trial session, without the element of stress and fear.

\section{Persistence of amnesia after reactivation}

The amnesia seen in the reminded rats in the present study does not appear to be transient; it endures for at least $48 \mathrm{~h}$ after the reminder-drug treatment. There have, however, been two reports of transient memory deficits related to APV injection after reactivation. The investigators suggested that the treatment induces a temporary inaccessibility of the stored information, rather than a failure to reconsolidate. In a first study, Summers et al. (1997) studying the effects of central APV administration on reconsolidation of an aversive task in chicks, reported a transient loss of memory clearly evident 5 min post-reminder, lasting at least 180 min post-reminder and recovering by $24 \mathrm{~h}$. Another study reports that intrahippocampal administration of APV after retrieval produces a dip in performance on a second retrieval test, $24 \mathrm{~h}$ later, and a recovery on a subsequent test, $48 \mathrm{~h}$ after the administration of APV (Szapiro et al. 2003). Those authors suggested that the disruption observed $24 \mathrm{~h}$ after the reactivation represents a temporary retrieval deficit, not a lack of stored memory. Although we have not studied the persistence of the amnesia evaluating memory at intervals longer than $48 \mathrm{~h}$, the amnesia shown in our experiments is robust and persistent for at least $48 \mathrm{~h}$.

\section{Consolidation and reconsolidation: Neural circuits involved}

The injections of APV were made into the lateral ventricles, so we cannot ascertain the site of action of the drug. The model proposed by Tsien (Shimizu et al. 2000) puts emphasis on synaptic re-entry reinforcement occurring in a reactivated hippocampalcortical-hippocampal circuit, because the subjects of their experiments were mice with targeted deletion of the NMDA NR1 receptor gene in CA1 of the hippocampus. Moreover, the memory deficit was revealed by a hippocampal-dependent behavioral task, the Morris water maze. Day and Morris (2001), on the other hand, were unable to produce memory deficits in a similar task by pharmacological infusion of APV into the ventricles (icv) in rats during the same extended post-training time window used by Shimizu et al. (2000). This may be due to a failure of the drug administered in the lateral ventricles to reach a sufficient volume of hippocampal tissue to be effective in disrupting this highly hippocampal-dependent task (Shimizu et al. 2001). There is converging evidence that the odor discrimination task used in the present experiment does not rely on the hippocampus. Posttraining injection of APV, icv or into the medial frontal cortex, 
induces amnesia for this task, while injection into the hippocampus does not (Tronel and Sara 2003). This question can only be answered by making local injections into specific regions after reactivation treatments. Meanwhile, the present results using a nonhippocampal-dependent task suggest that the involvement of NMDA receptors in reconsolidation processes is not restricted to the hippocampal-cortical circuits, as already suggested by Shimizu et al. (2001).

In conclusion, the present results suggest a role for NMDA receptors in memory processes occurring long after initial consolidation, because blockade of these receptors just after a cued memory reactivation procedure induces amnesia for the odorreward association. The involvement of NMDARs in memory reconsolidation of this task provides additional support for the idea that reconsolidation might recapitulate some cellular events that occur in memory consolidation.

\section{Materials and Methods}

\section{Animals}

Forty-two male Sprague-Dawley rats (IFFA-Credo; 200-220 g) were housed in pairs in a temperature- and light-controlled vivarium, on a 12-h light/12-h dark cycle. Rats were weighed and handled daily and had free access to food and water except during the pre-training, training, reactivation, and test days, when food was restricted to maintain body weight at $\sim 90 \%$ of freely feeding weight.

\section{Surgery}

Under pentobarbital anesthesia $(60 \mathrm{mg} / \mathrm{kg}$, intraperitoneal), stainless steel guide cannulae (14-mm-long, 24-gauge) aimed just dorsal to the lateral ventricles were implanted bilaterally according to standard stereotaxic procedures. The following coordinates were used: $1 \mathrm{~mm}$ posterior to Bregma, $\pm 2 \mathrm{~mm}$ lateral to the midline, $3 \mathrm{~mm}$ ventral to the surface of the skull (Paxinos and Watson 1986). A thin stainless-steel wire was placed inside each cannula to prevent blockage.

\section{Pharmacological treatment}

The fine wire was removed and replaced by the injector cannula (15-mm-long, 36-gauge) connected to a 5- $\mu \mathrm{L}$ Hamilton syringe by polyethylene tubing. The injector cannula protruded $1 \mathrm{~mm}$ beyond the tip of the guide cannulae to reach the lateral ventricles. The injection volumes were $3 \mu \mathrm{L}$ per side, delivered over a period of $1 \mathrm{~min}$ while the rat was gently held in the hand of the experimenter. One minute was allowed for diffusion of the drug before removing the injector. Control rats were injected with a vehicle solution $\left(0.9 \mathrm{~g}\right.$ of $\mathrm{NaCl}, 4.5 \mathrm{~mL}$ of $\mathrm{NaHPO}_{4}, 0.2 \mathrm{M}$ and $0.95 \mathrm{~mL}$ of $\mathrm{NaH}_{2} \mathrm{PO}_{4}, 2 \mathrm{H}_{2} \mathrm{O}, 0.2 \mathrm{M}$ in $100 \mathrm{~mL}$ of $\mathrm{dH}_{2} \mathrm{O}$ ). Experimental rats were injected bilaterally with 40 nmoles DL-APV per side $(2.5 \mu \mathrm{g} / \mu \mathrm{L}$, Sigma-Aldrich) dissolved in the vehicle solution.

\section{Apparatus}

The training apparatus and behavioral procedure have been described in detail elsewhere (Tronel and Sara 2002). A square box of opaque plastic $(60 \times 60 \times 40 \mathrm{~cm})$ contained sponges $(6 \times 7$ $\times 2 \mathrm{~cm}$ ) with a 2-cm-diameter hole cut into the center, placed in glass slide-holders of the same size. The food reinforcement was placed at the bottom of the opening in the sponge so the rat had to poke its snout into the hole (nose poke) to obtain the reward, chocolate rice crispy breakfast cereal (Chocopops, Kellogg's, France). On the first trial, four Chocopops were also placed on the corners of the sponge impregnated with the target odor, as well as in the hole. The sponges with the nontarget odors did not contain food. Sponges were placed in three corners of the box, and the position of each odor within the box was changed for each trial according to a previously determined protocol. The actual set of sponges was changed between trials as well, to preclude identification based on visual cues. Sponges were impreg- nated with $15 \mu \mathrm{L}$ of essence on each corner. Odors used were almond, mint, and lemon; previous experiments showed that rats did not show any particular preference for, or aversion to, any of these odors. In this experiment it was the almond odor that was reinforced.

A video camera was fixed above the apparatus, and the rat was observed on a video monitor in the same room. The path of the rats during the search was recorded using a video tracking system (Ethovision; Noldus).

\section{Experimental design and behavioral procedures}

Rats were handled and weighed daily during the recovery period ( $1 \mathrm{wk})$. The experiment began with $2 \mathrm{~d}$ of pre-training to familiarize the reinforcement and the experimental box. The first day rats were given free access to the reward for $20 \mathrm{~min}$ in a neutral cage; the second day, for $10 \mathrm{~min}$ in the same neutral cage and then placed $10 \mathrm{~min}$ in the experimental box without the sponges.

\section{Acquisition session}

Training was carried out the next day in a single three-trial session in the same room as the pre-training session. Cage mates were kept in a holding cage, and one rat was introduced into the experimental box, in the corner without a sponge, head toward the wall. There was a 5-min ceiling for the rat to find and consume the reinforcement. Intertrial intervals were 2-5 min. The spatial configuration of the sponges was changed between trials, and the reinforcement was always associated with the same odor. Latency before a correct response (nose poke into the reinforced sponge) and errors (nose poke into incorrect sponges or sniffing the target odor not followed by a nose poke) were noted. Rats that did not nose poke within 1 min or made more than 1 error at the third trial were eliminated from the experiment $(n=6)$. Animals were assigned to treatment groups according to their performance during training, in order to have three groups with no differences in number of errors or latencies at the end of acquisition.

\section{Reactivation session}

Twenty-four hours after acquisition, rats received a reactivation session, a 90-sec exposure to the reinforced odor in the holding cage, in the same room where the learning took place. Immediately after reactivation, rats were injected with vehicle (REACTVEH group, $n=7$ ) or with APV (REACT-APV group, $n=6$ ), in a quiet room adjacent to the experimental room.

\section{Retention test}

Forty-eight hours after the reactivation session, rats were tested for retention and relearning using the same procedure, except that the first test trial was not reinforced and there were four trials. The first trial served as a direct a measure of memory of the previous training. Since the first trial was not reinforced, the second trial reflected resistance to extinction, which is considered an indirect, but more sensitive measure of retention. The last two trials were an index of the rats' relearning ability. Latency to nose poke as well as errors of commission (nose poke to a nontarget odor) and omission (failure to nose poke after sniffing the sponge containing the target odor) were scored by an experimenter blind to the treatment groups.

One complementary experiment replicated the effects of APV on a reactivated memory and controlled for the specificity of the effects by adding a group that was not subjected to a reactivation session and injected with APV. Two groups of rats were trained and subjected to a reactivation session as in the preceding experiment and injected with vehicle (REACT-VEH group, $n=6$ ) or APV (REACT-APV group, $n=9$ ). A control group was not subjected to a reactivation session, and $24 \mathrm{~h}$ after the acquisition session received an injection of APV, in the vivarium (nonREACT-APV group $n=5$ ). Rats of all groups were tested $48 \mathrm{~h}$ after the injections, in the same conditions as in the previous experiment. 
After the retention session, rats were anesthetized with an overdose of Pentobarbital and received intracerebroventricular (icv) injections of methyl blue. Brains were removed and stored in $10 \%$ formalin. Brains were sectioned at $60 \mu \mathrm{m}$ thickness and examined by light microscopy to visually verify the placement of the cannulae and the presence of the dye in the ventricles. Only the behavioral data from animals with the cannulae located in the intended site and successful control injections were considered. Three rats were excluded from the statistical analyses.

\section{Data analysis}

Latency to nose poke and number of errors were taken as the performance measures. Acquisition scores for the entire data set, in terms of latencies and errors, were submitted to an analysis of variance for repeated measures (ANOVA). There was no effect of replication; therefore data were pooled for subsequent analysis.

For the statistical analysis the scores for the last acquisition trial and the average for the two retention trials and the two relearning trials were considered. Data were submitted to a $3 \times 3$ analysis of variance with repeated measures (between factor, group: REACT-VEH, REACT-APV, nonREACT-APV; within factor: last training trial, test, and relearning).

\section{Acknowledgments}

M.T.-G. was a recipient of a post-doctoral research grant from the Fyssen Fondation; J.L. was supported by a grant from the Volkswagenstifftung (to S.J.S.) and S.T. by the Fondation pour la Recherche Medicale. The research was funded by CNRS (UMR 7102). We thank Yves Moricard for histology and help in preparation of the manuscript.

\section{References}

Anokhin, K.V., Tiunova, A.A., and Rose, S.P. 2002. Reminder effects-Reconsolidation or retrieval deficit? Pharmacological dissection with protein synthesis inhibitors following reminder for a passive-avoidance task in young chicks. Eur. J. Neurosci. 15: $1759-1765$.

Bartlett, F.C. 1932. Remembering. A study in experimental and social psychology. University Press, Cambridge, UK.

Day, M. and Morris, R.G. 2001. Memory consolidation and NMDA receptors: Discrepancy between genetic and pharmacological approaches. Science 293: 755.

Debiec, J., LeDoux, J.E., and Nader, K. 2002. Cellular and systems reconsolidation in the hippocampus. Neuron 36: 527-538.

Dudai, Y. 2004. The neurobiology of consolidations, or, how stable is the engram? Annu. Rev. Psychol. 55: 51-86.

Hall, J., Thomas, K.L., and Everitt, B.J. 2001. Fear memory retrieval induces CREB phosphorylation and Fos expression within the amygdala. Eur. J. Neurosci. 13: 1453-1458.

James, W. 1890. The principles of psychology. Henry Holt, New York.

Kelly, A., Laroche, S., and Davis, S. 2003. Activation of mitogen-activated protein kinase/extracellular signal-regulated kinase in hippocampal circuitry is required for consolidation and reconsolidation of recognition memory. J. Neurosci. 23: 5354-5360.
Kida, S., Josselyn, S.A., de Ortiz, S.P., Kogan, J.H., Chevere, I., Masushige, S., and Silva, A.J. 2002. CREB required for the stability of new and reactivated fear memories. Nat. Neurosci. 5: 348-355.

Lewis, D., Bregman, N.J., and Mahan, J. 1972. Cue-dependent amnesia in rats. J. Comp. Physiol. Psychol 81: 243-247.

Milekic, M.H. and Alberini, C.M. 2002. Temporally graded requirement for protein synthesis following memory reactivation. Neuron 36: 521-525.

Nader, K., Schafe, G.E., and LeDoux, J.E. 2000. Fear memories require protein synthesis in the amygdala for reconsolidation after retrieval. Nature 406: 722-726.

Paxinos, G. and Watson, C. 1986. The rat brain in stereotaxic coordinates. Academic Press, New York.

Pedreira, M.E., Perez-Cuesta, L.M., and Maldonado, H. 2002. Reactivation and reconsolidation of long-term memory in the crab Chasmagnathus: Protein synthesis requirement and mediation by NMDA-type glutamatergic receptors. J. Neurosci. 22: 8305-8311.

Przybyslawski, J. and Sara, S.J. 1997. Reconsolidation of memory after its reactivation. Behav. Brain Res. 84: 241-246.

Przybyslawski, J., Roullet, P., and Sara, S.J. 1999. Attenuation of emotional and nonemotional memories after their reactivation: Role of $\beta$-adrenergic receptors. J. Neurosci. 19: 6623-6628.

Roullet, P. and Sara, S. 1998. Consolidation of memory after its reactivation: Involvement of $\beta$-noradrenergic receptors in the late phase. Neural Plast. 6: 63-68.

Sangha, S., Scheibenstock, A., and Lukowiak, K. 2003. Reconsolidation of a long-term memory in Lymnaea requires new protein and RNA synthesis and the soma of right pedal dorsal 1. J. Neurosci. 23: $8034-8040$.

Sara, S.J. 2000. Retrieval and reconsolidation: Toward a neurobiology of remembering. Learn. Mem. 7: 73-84.

Shimizu, E., Tang, Y.P., Rampon, C., and Tsien, J.Z. 2000. NMDA receptor-dependent synaptic reinforcement as a crucial process for memory consolidation. Science 290: $1170-1174$.

Shimizu, E., Tang, Y.P., Rampon, C., Feng, R., and Shrom, D. 2001 Memory consolidation and NMDA receptors: Discrepancy between genetic and pharmacological approaches. Science 293: 755.

Summers, M.J., Crowe, S.F., and Ng, K.T. 1997. Administration of DL-2-amino-5-phosphonovaleric acid (AP5) induces transient inhibition of reminder-activated memory retrieval in day-old chicks. Brain Res. Cogn. Brain Res. 5: 311-321.

Szapiro, G., Vianna, M.R., McGaugh, J.L., Medina, J.H., and Izquierdo, I. 2003. The role of NMDA glutamate receptors, PKA, MAPK, and CAMKII in the hippocampus in extinction of conditioned fear. Hippocampus 13: 53-58.

Taubenfeld, S.M., Milekic, M.H., Monti, B., and Alberini, C.M. 2001. The consolidation of new but not reactivated memory requires hippocampal C/EBPß. Nat. Neurosci. 4: 813-818.

Tronel, S. and Sara, S.J. 2002. Mapping of olfactory memory circuits: Region-specific c-fos activation after odor-reward associative learning or after its retrieval. Learn. Mem. 9: 105-111.

. 2003. Blockade of NMDA receptors in prelimbic cortex induces an enduring amnesia for odor-reward associative learning. $J$. Neurosci. 23: 5472-5476.

Wittenberg, G.M. and Tsien, J.Z. 2002. An emerging molecular and cellular framework for memory processing by the hippocampus. Trends Neurosci. 25: 501-505.

Received April 30, 2004; accepted in revised form October 12, 2004.

\section{Learning \& Memory}




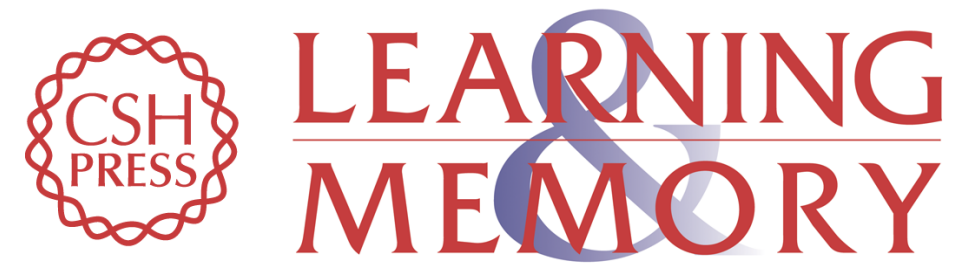

\section{Reconsolidation after remembering an odor-reward association requires NMDA receptors}

Meritxell Torras-Garcia, Julien Lelong, Sophie Tronel, et al.

Learn. Mem. 2005, 12:

Access the most recent version at doi:10.1101/lm.80905

References This article cites 23 articles, 8 of which can be accessed free at:

http://learnmem.cshlp.org/content/12/1/18.full.htmI\#ref-list-1

License

Email Alerting Receive free email alerts when new articles cite this article - sign up in the box at the Service top right corner of the article or click here. 